\title{
VARIACIÓN GEOGRÁFICA EN PHRYNOSOMA ORBICULARE (SAURIA: PHRYNOSOMATIDAE): ANÁLISIS DE LAS SUBESPECIES
}

\section{RUTH MORENO-BARAJAS ${ }^{1}$, FELIPE RODRÍGUEZ-ROMERO ${ }^{1 *}$, ALMA VELÁZQUEZ-RODRÍGUEZ ${ }^{1}$ \& ANDRÉS ARAGÓN-MARTÍNEZ ${ }^{2}$}

${ }^{1}$ Centro de Investigación en Recursos Bióticos. Universidad Autónoma del Estado de México. Campus El Cerrillo, Piedras Blancas, Carretera Toluca - Ixtlahuaca; Km 15.5. Toluca, Edo. de México. CP. 52000.

${ }^{2}$ Facultad de Medicina Veterinaria y Zootecnia. Universidad Autónoma del Estado de México. Campus El Cerrillo, Piedras Blancas, Carretera Toluca - Ixtlahuaca; km 15.5 Toluca, Edo. de México. CP. 52000.

*Autor corresponsal:<fjrr@uaemex.mx>,< alin_ruth@hotmail.com>, <almaver22@hotmail.com>, $<$ aam@uaemex.mx>.

Moreno-Barajas, R., Rodríguez-Romero, F., Velázquez-Rodríguez, A. \& Aragón-Martínez, A. 2013. Variación geográfica en Phrynosoma orbiculare (Sauria: Phrynosomatidae): análisis de las subespecies. Acta Zoológica Mexicana (n. s.), 29(1): 129-143.

RESUMEN. Para el complejo Phrynosoma orbiculare se han descrito seis subespecies que se distribuyen desde el norte del país, hasta el sureste de la planicie Mexicana, desde su descripción, el estado taxonómico de las subespecies de $P$. orbiculare presentan una serie de discordancias. Por lo que el objetivo del presente estudio fue evaluar la variación morfológica de las subespecies descritas, utilizando análisis de morfometría lineal y geométrica combinados con análisis de componentes principales y de correlación canónica. Los análisis con morfometría lineal no muestran una clara definición, sin embargo con la morfometría geométrica, mediante el análisis de variación canónica se obtuvieron diferencias significativas para tres subespecies, $P$. o orientale, $P$. o. durangoensis y $P$. o. boucardi. La gradilla de deformación muestra que la variación se encuentra principalmente hacia la región de la punta del hocico y de la corona. P. o. orientale se distribuye en la Sierra Madre Oriental y P. o. durangoensis en la Sierra Madre Occidental ambas se encuentran aisladas por la Meseta Central y su aislamiento data desde la vicarianza del Neogeno, por lo que si estas subespecies se encuentran aisladas pueden ser consideradas como entidades diferentes ya que no hay flujo génico entre ellas.

Palabras clave: Phrynosoma orbiculare, morfometría geométrica, variación, subespecies, estadística multivariada.

Moreno-Barajas, R., Rodríguez-Romero, F., Velázquez-Rodríguez, A. \& Aragón-Martínez, A. 2013. Geographical variation in Phrynosoma orbiculare (Sauria: Phrynosomatidae): subspecies analysis. Acta Zoológica Mexicana (n. s.), 29(1): 129-143.

Recibido: 03/02/2012; aceptado: 12/11/2012. 
Moreno-Barajas et al.: Variación geográfica en Phrynosoma orbiculare

ABSTRACT. For the Phrynosoma orbiculare complex six subspecies have been described that are distributed from the north to the southeast of the Mexican Plateau. Since, the description of the group, the taxonomic status of the subspecies of $P$. orbiculare has been debated. The objective of this study was to assess the morphological variation of the subspecies described; using analysis of linear and geometric morphometrics combined with principal component analysis and canonical correlation. The linear morphometric analysis showed a clear definition, but with geometric morphometrics, using canonical variance analysis, significant differences were obtained for three subspecies, $P$. o. orientale, $P$. o. durangoensis and $P$. o. boucardi. The thin plate spline showed that main deformation is in the snout and horns combined. Phrynosoma. o. orientale is distributed in the Sierra Madre Oriental and P. o. durangoensis in the Sierra Madre Occidental. Both subspecies are isolated by the Central Mexican Plateu and diverged as a result of Neogene vicariance. So if subspecies are isolated can be considered as separate entities since there is no gene flow between them.

Key words: Phrynosoma orbiculare, geometric morphometrics, variation, subspecies, multivariate statistics.

\section{INTRODUCCIÓN}

Las subespecies tienen una historia amplia en la taxonomía, en la época de Linneo eran llamadas variedades y no había distinción entre variedades individuales y geográficas, algunos autores como Esper, utilizaron la palabra subespecie por primera vez para designar variedades geográficas (Mayr 1982). Posteriormente, a finales del siglo XIX las subespecies adquirieron un significado biológico dual; por una parte, se consideraron como las especies incipientes que describió Darwin y que se encuentran en proceso de especiación y, por otra, autores como Gloger, Bergman, y J. A. Allen, consideraron que eran evidencia de la respuesta adaptativa de los organismos a condiciones climáticas tanto de poblaciones aisladas, como de poblaciones de amplia distribución (Mayr 1982).

Entre los años de 1920 a 1940 no hubo un cambio significativo en la descripción de las subespecies, ya que la asignación de esta categoría seguía siendo arbitraria, lo cual generó un abuso en la denominación trinomial, debido a que cualquier ligera variación en algún caracter era suficiente para utilizar la nomenclatura subespecífica (Wilson \& Brown 1953, Inger 1961, Mayr 1982). Por lo tanto, se propuso una metodología equivalente a la que se utilizaba para describir especies, como la variación geográfica y zonas de contacto primario y secundario, para justificar la descripción de nuevas subespecies (Mayr 1982). Con base en criterios tanto morfológicos como geográficos, las subespecies se definieron como miembros de las especies politípicas y no como organismos con ligeras variaciones en las poblaciones locales (O'Neill 1982). En poblaciones alopátricas donde la distribución es clara y, en situaciones en las que hay contacto secundario entre ellas y las zonas de intergradación son relativamente estrechas, pueden utilizarse las descripciones subespecíficas como un concepto útil de discusión en los procesos evolutivos de especiación (Monroe 1982).

Dentro de los conceptos modernos, las subespecies no sólo son unidades taxonómicas, también son unidades evolutivas, principalmente cuando están geográfica- 
mente aisladas, ya que en los procesos evolutivos de adaptación y selección natural, la variación es fundamental y cuando en una población se estabiliza la variabilidad por selección natural ésta puede representar el primer paso en la formación de especies, hasta que eventualmente queden aisladas reproductivamente (Monroe 1982, O'Neill 1982). En este sentido el concepto de subespecie puede proporcionar información importante sobre los mecanismos de especiación, de manera que puede ser válido siempre y cuando esté relacionado con los procesos evolutivos de dichos mecanismos (O’Neill 1982).

En las investigaciones recientes, el enfoque que se presenta va más allá del debate del concepto de subespecie, ya que las descripciones subespecíficas se utilizan de manera amplia; con base en esto, la pregunta principal es ¿Qué hacer con ellas? (Manier 2004). Un primer paso es evaluar los caracteres diagnósticos de las subespecies existentes, para ello se han utilizado diversos métodos que permiten evaluar desde otra perspectiva los caracteres morfológicos diagnósticos. Algunos de ellos están circunscritos en la morfometría lineal y otros en la morfometría geométrica, ambas en combinación con la estadística multivariada (Smith 1997). Estos análisis permiten, de acuerdo con algunos autores, redefinir subespecies o asignar nuevos individuos a un grupo subespecífico; pero además, algunos de ellos sugieren cambios en el nivel de clasificación taxonómica de los organismos, es decir, elevar las subespecies a la categoría de especie (Manier 2004, Montanucci 2004, Rastegar-Pouyani 2005, Bastos-Silveria 2007).

Phrynosoma orbiculare (Linnaeus 1789) es un lacertilio que se distribuye desde el norte del país hasta el sureste de la planicie Mexicana (Méndez de la Cruz et al. 2002) (Fig. 1). Habita en zonas semidesérticas y en zonas de clima templado en altitudes que van desde los 1,371 hasta los 3,352 metros (Montanucci 1987). Para esta especie se han descrito seis subespecies, $P$. o. bradti (Dunn 1936) que se distribuye en el oeste de Chihuahua; $P$. o. durangoensis (Boulenger 1885) registrada para el suroeste de Durango, Jalisco y Zacatecas; P. o. orientale (Garman 1887) en los estados de San Luis Potosí, Tamaulipas y Nuevo León; P. o. cortezii (Duméril y Bibron 1837) que se distribuye en el sur de Zacatecas, zonas altas de Jalisco, Hidalgo, Puebla y Veracruz; P. o. orbiculare (Linneaus 1758) en el Distrito Federal, Tlaxcala y Estado de México (Horowitz, 1955); y P. o. boucardi que se distribuye en el norte de los estados de Hidalgo y Querétaro (Dúmeril y Bocourt 1870).

Desde su descripción, las subespecies de $P$. orbiculare presentan una serie de discordancias morfológicas y taxonómicas (Montanucci 1979) y, sin embargo, los estudios reportados son escasos. Uno de los primeros que se planteó para reevaluar las subespecies de $P$. orbiculare y su variación geográfica, se realizó mediante el análisis de caracteres morfológicos como la longitud de los cuernos occipitales y temporales, longitud de la cola, ancho y largo de la cabeza, así como pruebas estadísticas univariadas (Horowitz 1955). 


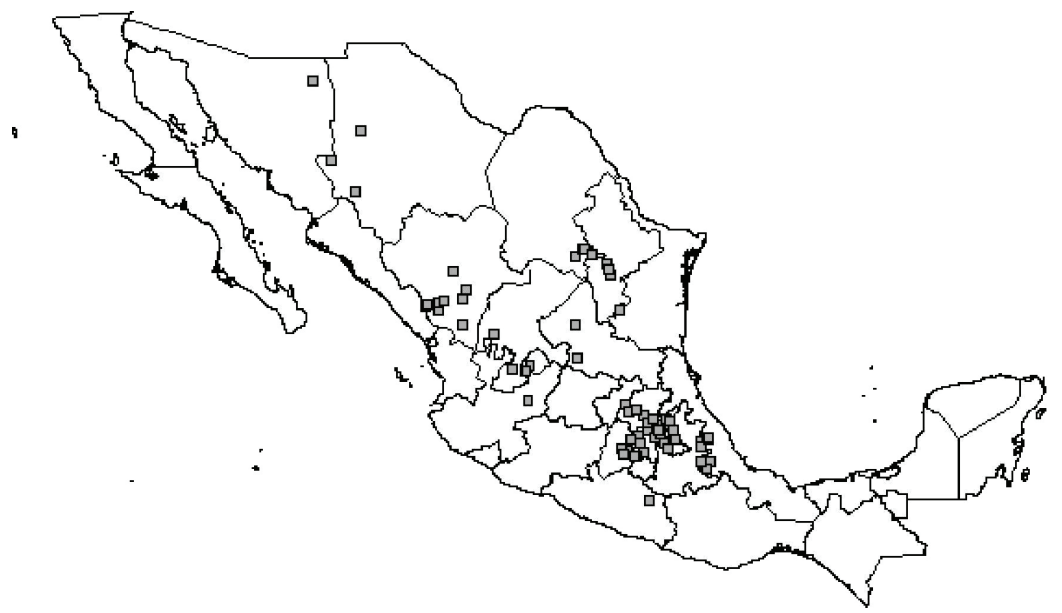

Figura 1. Distribución geográfica del complejo Phrynosoma orbiculare.

Debido a que para el complejo $P$. orbiculare no hay registros de estudios donde se haya efectuado una evaluación de la situación taxonómica con análisis morfométricos más precisos como los análisis estadísticos multivariados, el objetivo del presente estudio fue evaluar la variación morfológica de las subespecies validadas para el complejo $P$. orbiculare mediante el uso de análisis de morfometría líneal y geométrica, combinadas con análisis de componentes principales y de correlación canónica.

\section{MATERIAL Y MÉTODOS}

Se examinaron un total de 65 ejemplares, 42 provenientes del Museo "Alfonso L. Herrera" de la Facultad de Ciencias de la Universidad Nacional Autónoma de México, 13 de la Colección Nacional de Anfibios y Reptiles del Instituto de Biología, UNAM, seis de la Colección Herpetológica de la Facultad de Ciencias Biológicas de la Universidad Autónoma de Nuevo León, así como cuatro individuos colectados en la localidad de Perote, Veracruz (N19³2'31.2"; W97¹6'38.2") (Anexo). Para la revisión taxonómica se consideró la propuesta de Horowitz (1955) el primer autor que investigó la variación geográfica de Phrynosoma orbiculare en México.

Los ejemplares analizados son representativos de diversas localidades que cubren el intervalo de distribución del grupo $P$. orbiculare (Fig. 2).

Análisis Morfométricos. Las variables morfológicas se analizaron mediante morfometría tradicional (Marcus 1990) y morfometría geométrica de configuraciones de puntos homólogos (landmarks) (Bokstein 1991). Para el análisis con distancias lineales se tomaron fotografías digitales a cada uno de los ejemplares con una cámara 


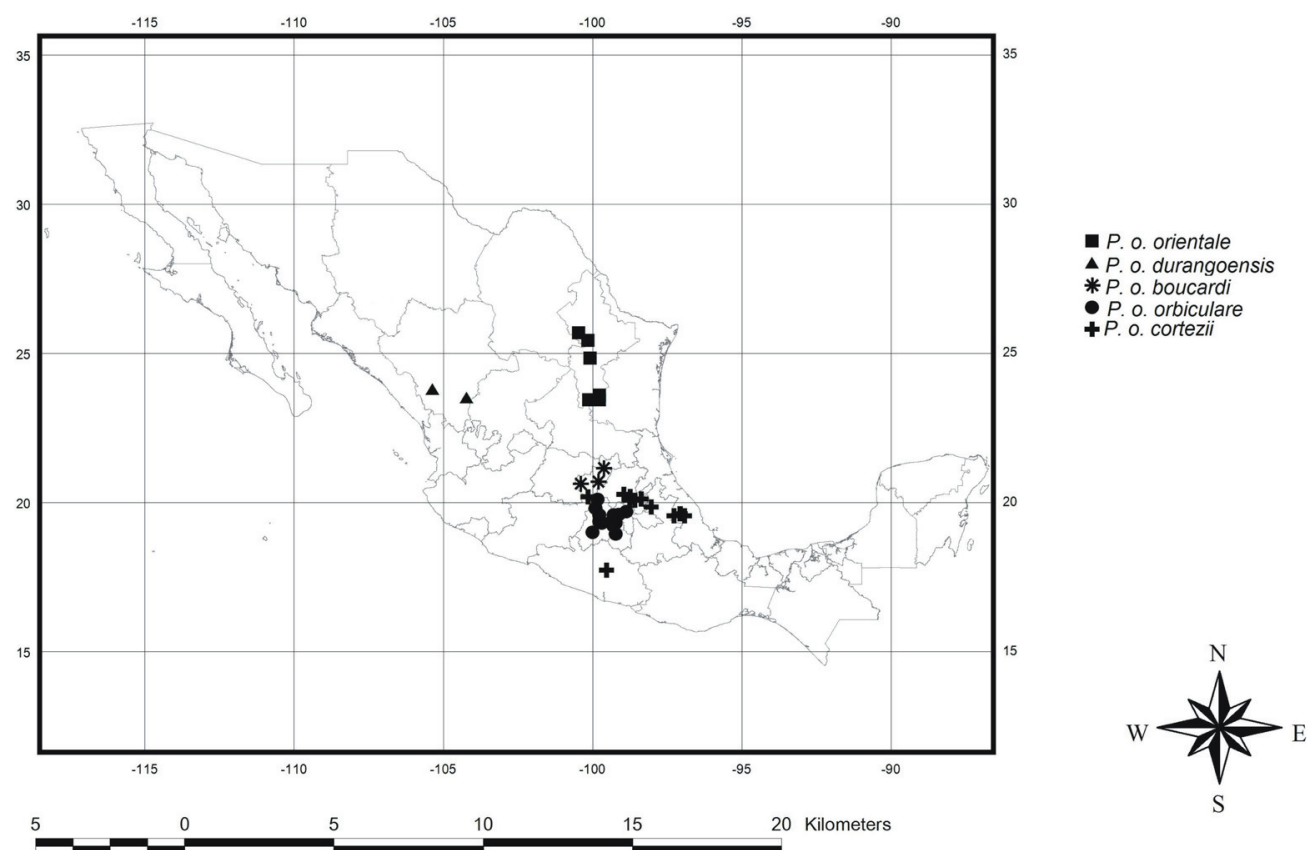

Figura 2. Mapa que muestra la distribución de las localidades analizadas para las subespecies de Phrynosoma orbiculare.

Sony DSC-F828 a una resolución de 7 megapixeles y para calibrar cada imagen, se colocó una escala milimétrica; posteriormente se midieron los siguientes caracteres morfométricos con el programa ImageJ 1.38r (Rasband, W): ancho de la cabeza (ANCAB), largo de la cabeza (LCAB), longitud de los cuernos occipitales (LCOCC), longitud de los cuernos temporales (LCTEM), longitud de las escamas superciliares (LESUP), los cuales se digitalizaron y midieron con el mismo programa, adicionalmente se midió la longitud hocico-cloaca (LHC) para estandarizar el resto de las variables, mediante la obtención de los residuos para cada uno de los caracteres en el análisis de regresión lineal. La elección de estas variables se justifica con base en que son caracteres que se utilizaron como diagnósticos en la determinación de las subespecies (Horowitz 1955). Posteriormente se realizó un análisis de componentes principales para evaluar los caracteres con los eigenvalores más altos, así como para analizar el porcentaje de variación explicado.

Para el análisis de morfometría geométrica, se seleccionaron imágenes individuales de la vista dorsal del cráneo de cada organismo, cada una de ellas con una escala de referencia de $1 \mathrm{~mm}$. Con el fin de registrar adecuadamente la información del diseño del cráneo, se colocaron 33 puntos homólogos (landmarks) con el Programa TPS- 
DIG de la serie TPS (Zelditch et al. 2004), el cual genera un par de coordenadas (x, y) para cada marca registrada. Los landmarks se colocaron en los siguientes sitios diagnósticos: punta del hocico, narinas, borde de los ojos, base y punta de los cuernos temporales y occipitales, base y punta de las escamas superciliares y ojo pineal (Fig. 3).

Con el Programa Paleontological Statistics "PAST" (Harmmer \& Harper 1999) se realizó la superimposición por procrustes para eliminar el efecto de la ubicación, orientación y el tamaño y así evaluar la variación en la forma. Las placas delgadas de deformación se construyeron aplicando la función de interpolación thin plate spline (Bokstein 1991), a partir de la cual se pueden obtener los partial warps scores donde las columnas de la matriz de peso (W) representan los valores para cada organismo. Estos valores pueden utilizarse como nuevas variables de forma en los análisis estadísticos multivariados para comparar las diferencias entre las poblaciones (Zelditch et al. 2004).

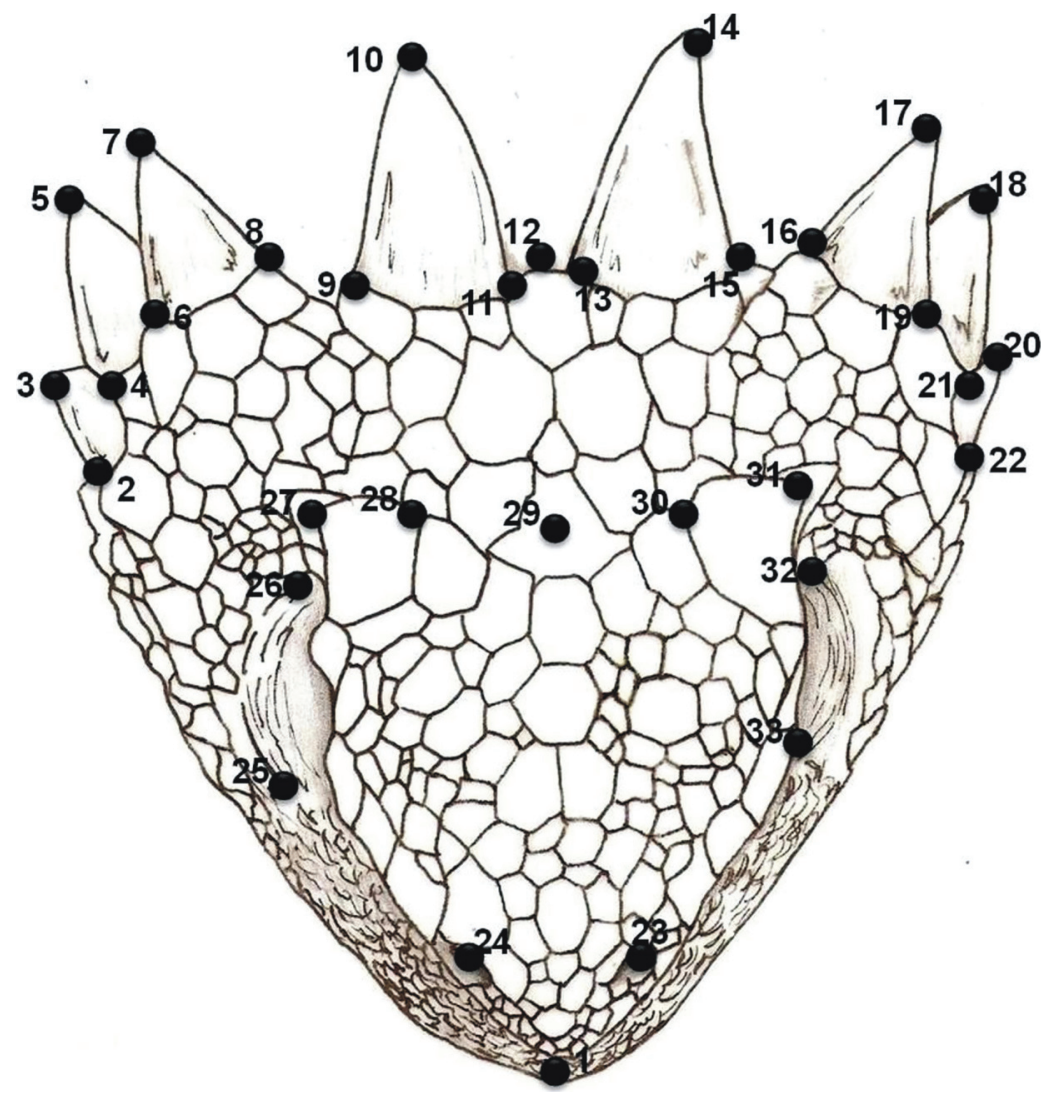

Figura 3. Ubicación de los landmarks en el cráneo de Phrynosoma orbiculare. 
En el programa PAST también se realizó un análisis de componentes principales (ACP) para evaluar la distribución de las poblaciones de acuerdo con las distancias entre los landmarks de cada organismo, este análisis también permite obtener la "gradilla de deformación" para cada una de las poblaciones de interés.

Para evaluar las diferencias estadísticas en cada población, se realizó un análisis de variación canónica (CVA) que permite encontrar diferencias significativas entre grupos conocidos, mediante una prueba de análisis de varianza multivariado (MANOVA).

Con el fin de determinar a qué grupos corresponden las diferencias, se realizaron comparaciones pareadas usando las distancias euclideanas entre los promedios de los mínimos cuadrados, el cual es un procedimiento de permutaciones al azar con los residuales del modelo lineal, cuya ventaja es que permite encontrar las diferencias morfológicas entre las especies de una manera consistente, sin olvidar la covariación de los rasgos múltiples (Adams 2007). Finalmente mediante un análisis de funciones discriminantes, se determinó el total y el porcentaje de organismos correctamente clasificados para cada población.

\section{RESULTADOS}

Análisis morfométrico con medidas lineales. Inicialmente se analizó el dimorfismo sexual de los ejemplares para cada población mediante la prueba de ANOVA, los resultados muestran que no hay diferencias significativas entre sexos. De tal manera que el resto de las pruebas estadísticas se realizaron con machos y hembras en conjunto para cada población. El análisis de componentes principales con los ejemplares de las nueve localidades, muestra tres grupos que corresponden a las localidades de Veracruz (A), Querétaro (B) y Nuevo León (C) (Fig. 4). La distribución de los grupos es a lo largo del CP 2 que explica la separación de estas poblaciones con $21.79 \%$ de la variación lo que se atribuye principalmente a el ancho y largo de la cabeza, así como a la distancia entre las escamas superciliares que son las variables que tienen la mayor carga tanto positiva como negativa (Cuadro 1). Para el CP 1 todos los coeficientes son positivos, lo que indica que existe una correlación de este tipo entre todas las variables, por otro lado, la distancia entre las escamas superciliares y la longitud de la cabeza son las variables con los valores más altos. Sin embargo, estas variables no contribuyen a la separación de grupos (Cuadro 1).

Análisis con morfometría geométrica. Los resultados del análisis de componentes principales se muestran en una gráfica de distribución con los primeros dos componentes. Se observa que, a pesar del traslape de prácticamente todas las poblaciones, en el componente 2 existe una separación entre la población de Nuevo León y la de Querétaro, con un porcentaje de variación de 18.7 (Fig. 5). En la gradilla de deforma- 


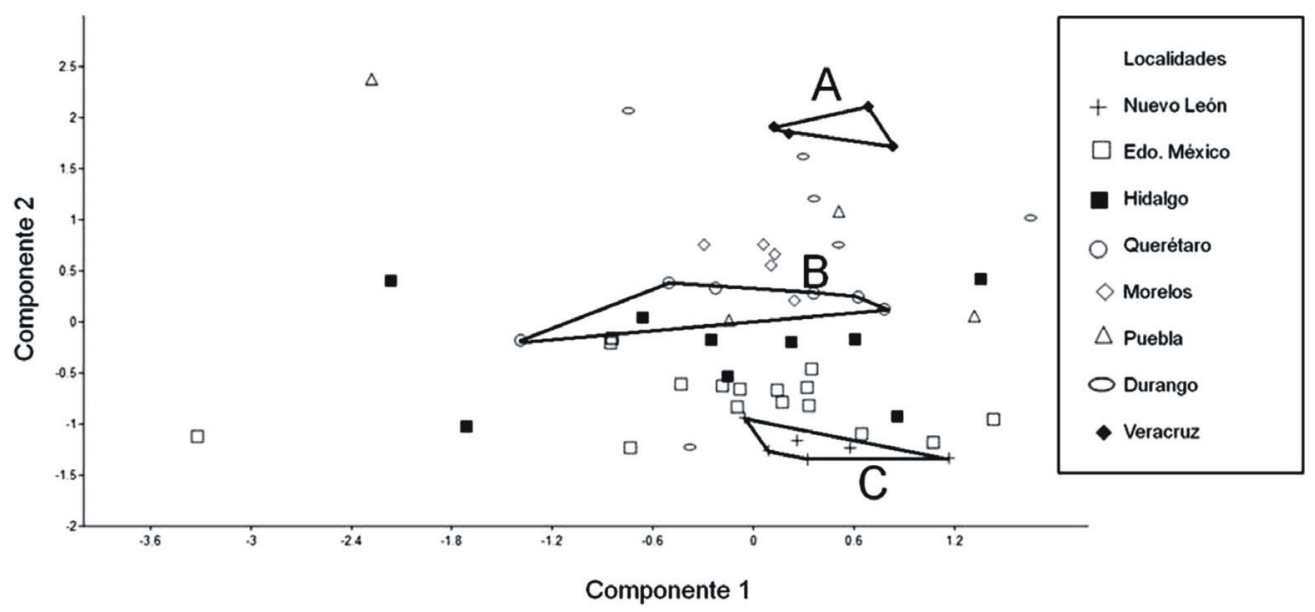

Figura 4. Gráfica de componentes principales. Muestra la formación de tres grupos a lo largo del CP2 Veracruz (A), Querétaro (B) y Nuevo León (C).

Cuadro 1. Carga de los primeros dos componentes principales, así como los eigenvalores, el porcentaje de varianza y el porcentaje de varianza acumulada para cada una de las variables analizadas en los ejemplares de Phrynosoma orbiculare de cada localidad.

\begin{tabular}{lll}
\hline Caracter & $\mathrm{CP} 1$ & $\mathrm{CP2}$ \\
\hline Largo-cabeza & 0.4185 & 0.562 \\
Ancho-cabeza & 0.321 & 0.593 \\
Longitud-cuernos occipitales & 0.198 & 0.0028 \\
Longitud-primer cuerno temporal & 0.035 & 0.14 \\
Longitud-segundo cuerno temporal & 0.030 & 0.145 \\
Longitud-escamas superciliares & 0.029 & 0.051 \\
Distancia-cuernos occipitales & 0.287 & -0.265 \\
Distancia-escamas superciliares & 0.772 & -0.467 \\
Eigenvalores & 9.8 & 4.3 \\
Porcentaje de varianza & 48.77 & 21.79 \\
Porcentaje de varianza acumulada & 48.77 & 70.56 \\
\hline
\end{tabular}

ción, para la población de Querétaro se observa una ligera reducción en los landmarks 1,24 y 23 , que corresponden a la punta del hocico, mientras que para la población de Nuevo León se observa un ligero ensanchamiento en los landmarks 2 a 22 ,que corresponden a la región de los cuernos temporales y occipitales.

En cuanto al análisis de variación canónica, los resultados indican que en el eje de las abscisas se separan cuatro poblaciones, Nuevo León, Durango, Querétaro y Vera- 


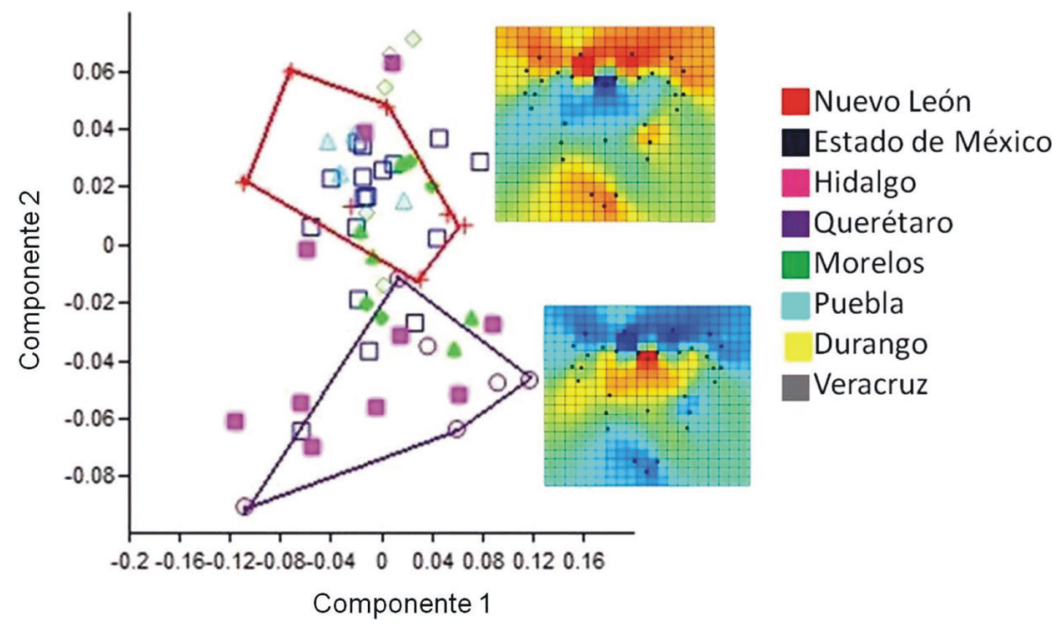

Figura 5. Análisis de componentes principales donde se muestra la separación de las poblaciones de Nuevo León y Querétaro, con la gradilla de deformación correspondiente. Las gradillas muestran un gradiente de color de azul a rojo, donde; el azul muestra la menor deformación y el rojo la mayor.

cruz, y en el eje de las ordenadas se separan dos poblaciones, Nuevo León y Puebla (Fig. 6). El MANOVA muestra que hay diferencias significativas entre las poblaciones de Nuevo León y Querétaro $(P=0.02)$ y Nuevo León y Durango $(P=0.024)$, además las distancias de Mahalanobis entre estas poblaciones presentan un valor de 17.18 y entre Nuevo León y Querétaro de 13.94, lo que indica que las diferencias son mayores entre poblaciones que dentro de ellas. En cuanto a las gradillas de deformación se puede apreciar que, para la población de Nuevo León hay un ensanchamiento en los landmarks 2 a 8 y 16 a 22 que corresponden a los cuernos temporales, así como en la región de las escamas superciliares que corresponden a los landmarks 27 a 31, mientras que en la región del hocico que corresponde a los landmarks 1, 23 y 24, la forma tiende a reducirse.

La placa de deformación para la población de Querétaro muestra un ensanchamiento principalmente hacia la región de los cuernos temporales así como de los cuernos occipitales en los landmarks 2 a 22 y, se estrecha hacia la parte del hocico en los landmarks 1, 23 y 24. Finalmente para la población de Durango la gradilla de deformación muestra que existe una constricción hacia la región media del cráneo, en la ubicación de los landmarks 25 a 33, así como en la región de los cuernos temporales, en la ubicación de los landmarks 2 a 8 y 26 a 22. Además para estas poblaciones, la tabla de clasificación muestra que para la población de Nuevo León $42.8 \%$ de los organismos están correctamente clasificados, mientras que para la población de Querétaro $66.6 \%$ y para la de Durango $60 \%$. 


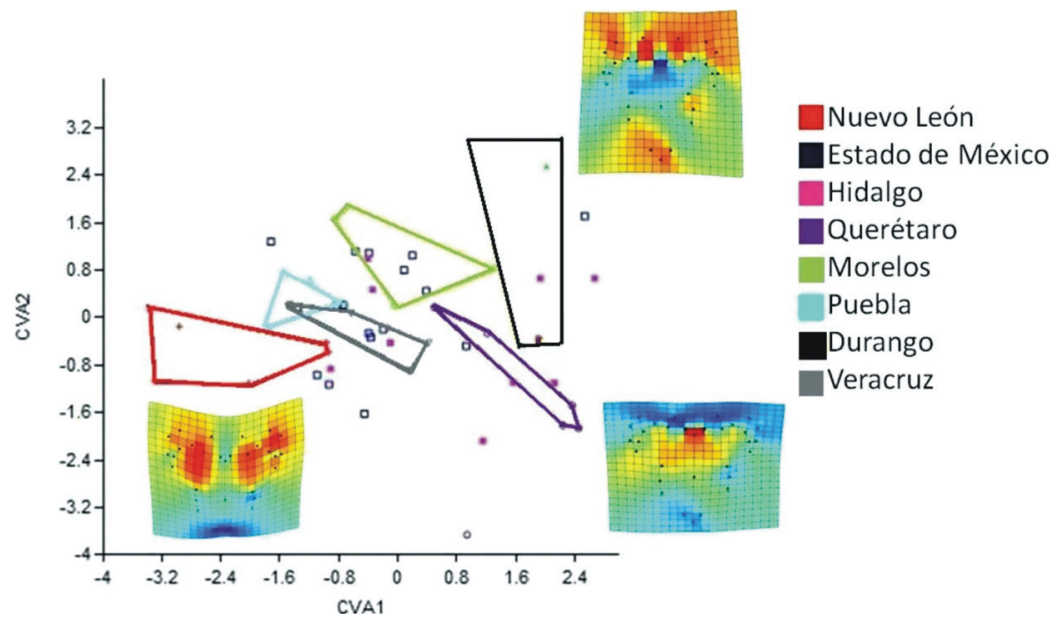

Figura 6. Análisis de variación canónica donde se muestran los grupos que se forman así como las gradillas de deformación para las poblaciones de Nuevo León, Querétaro y Durango.

\section{DISCUSIÓN}

Los estudios que se han realizado para definir la taxonomía del grupo $P$. orbiculare son escasos. Horowitz (1955) sugiere cinco subespecies y un intergrado, con base en análisis estadísticos univariados y caracteres como la longitud de los cuernos occipitales y temporales, número de escamas laterales y número de poros femorales. En un estudio reciente, se examinó mediante un análisis filogenético con DNA mitocondrial la relación de la distribución histórica y actual de $P$. orbiculare; los resultados muestran dos clados, el clado norte contiene a las poblaciones que se distribuyen en la Sierra Madre Occidental y el clado sur, contiene localidades que se encuentran tanto en el Sur de la Sierra Madre Oriental, como en la Faja Volcánica Transmexicana (Bryson et al. 2012). Sin embargo, no hay estudios recientes en los que se evalúe el estado taxonómico de la especie con herramientas morfométricas más precisas y mediante análisis estadísticos multivariados, que permitan complementar el trabajo de Bryson et al. (2012).

Los resultados del presente estudio muestran que las medidas lineales no permiten separar a las entidades biológicas, mientras que los análisis con morfometría geométrica, destacan claramente diferencias significativas entre grupos.

Las variaciones morfológicas, con medidas lineales y análisis univariados, entre las subespecies se observan principalmente en la longitud de la cola así como el largo y ancho de la cabeza. Se ha registrado que $P$. o. orbiculare tiene la cola proporcionalmente más corta que $P$. o. durangoensis y que $P$. o. orientale difiere en este caracter con respecto a $P$. o. bradti; por otro lado, en cuanto a la variación en el ancho y largo 
de la cabeza, se menciona que $P$. o. bradti difiere de $P$. o. cortezii y de $P$. o. orientale, ya que estas dos últimas subespecies se encuentran estrechamente relacionadas en cuanto a su distribución geográfica (Horowitz 1955). Sin embargo, en otros trabajos se muestran incongruencias entre caracteres diagnósticos como los poros femorales, los cuernos occipitales y los temporales, en subespecies como P. o. boucardi y $P$. o. orientale (Montanucci 1979 y Lawrence et al. 2002).

Aun cuando los análisis con morfometría tradicional no permitieron una clara separación entre los grupos, sí destacaron que entre las poblaciones de Veracruz, Nuevo León y Querétaro descritas como $P$. o. cortezii, $P$. o. orientale y $P$. o. boucardi, respectivamente, existe una tendencia a la separación de estos grupos a lo largo del CP2 con $21.96 \%$ de variación (Fig. 4) y el ancho y largo de la cabeza son los caracteres que contribuyen a la formación de estos grupos (Cuadro 1). A pesar de que el porcentaje de variación es bajo, los resultados de este análisis indican que existe una congruencia con lo reportado por Horowitz (1955) en cuanto al caracter que contribuye a la separación de $P$. o. cortezii y $P$. o. orientale, mientras que para $P$. o. boucardi hay una incongruencia en cuanto a los caracteres diagnósticos, ya que se reportó que esta subespecie se caracteriza por presentar cuernos occipitales erectos y escamas superciliares prominentes (Smith 1950). Sin embargo, con este análisis se encontró que es el ancho y largo de la cabeza el carácter que agrupa esta subespecie.

En cuanto al análisis de morfometría geométrica que se realizó con las nueve poblaciones, se observaron diferencias significativas apoyadas por las distancias de Mahalanobis entre las configuraciones geométricas de las poblaciones de Nuevo León - Durango $(P=0.02)$ y Nuevo León - Querétaro $(P=0.024)$, notándose que la mayor variación se encuentra en la forma del cráneo, tanto en la región de los cuernos occipitales y temporales, como en la punta del hocico (Fig. 6). A pesar de que con los dos métodos morfométricos, tradicionales y geométricos, no es posible hacer comparaciones directas (Manier 2004) sí es posible realizar algunas relaciones. Por ejemplo, el ancho y largo de la cabeza en el análisis de CP de medidas lineales es el caracter que presenta mayor peso y, con la placa fina de deformación resultante de la configuración de landmarks, se puede apreciar que, la variación se encuentra relacionada con este carácter; como se refiere a continuación para cada una de las poblaciones que mostraron diferencias significativas. En cuanto a la población de Querétaro, se describió a P. boucardi (Duméril \& Bocourt, 1870), cuyos caracteres diagnósticos son la presencia de cuernos occipitales erectos y escamas superciliares prominentes, no obstante, un estudio posterior explica que esta especie debe ser incluida como una subespecie de $P$. orbiculare ya que presenta características externas que la relacionan con $P$. o. cortezii y con $P$. o. orientale, por lo que puede ser un intergrado entre estas dos (Montanucci 1979). Como ya se mencionó anteriormente, la configuración geométrica para $P$. o. boucardi muestra diferencias significativas con respecto a $P$. o. orientale y no así con $P$. o. cortezii, y la gradilla de deformación muestra que la variación con- 
siste en que hay un ensanchamiento hacia la región de los cuernos temporales, pero no se observa deformación en las escamas superciliares como lo sugieren los caracteres diagnósticos. La distribución de $P$. o. boucardi se encuentra hacia el norte de los estados de Querétaro e Hidalgo, cuya región biogeográfica corresponde a la parte sur de la Meseta Central (MC), mientras que la distribución de $P$. o. cortezii es hacia algunas zonas de la Faja Volcánica Transmexicana (FVT), Meseta Central y Sierra Madre Oriental (SMO), de acuerdo con las poblaciones analizadas en este estudio.

Montanucci (1979), registra que $P$. o. boucardi se distribuye en localidades intergrado entre el área de distribución oriental para $P$. o. cortezii de tal manera que pueden existir algunas zonas de contacto entre las poblaciones de estas subespecies, esta puede ser una razón por la cual, no se encontraron diferencias significativas, aunque por otro lado, si se encontraron grupos definidos para las poblaciones de Puebla y Veracruz que corresponden a $P$. o. cortezii. Puede ser que, a pesar de la presencia de algunas barreras geográficas como el Río Moctezuma en Querétaro, aún no ocurre aislamiento completo, de tal manera que los resultados de este trabajo coinciden con lo reportado por Montanucci (1979), quien menciona que $P$. o. boucardi debe ser considerada una subespecie de $P$. orbiculare y no como especie incipiente. En las subespecies que se solapan en su distribución, la variación que se observó puede ser resultado de la presencia de híbridos, por lo tanto estas no pueden ser consideradas como entidades distintas (Smith 1942). También existe la posibilidad de que, con base en su distribución histórica, estas dos poblaciones permanecieron aisladas con el surgimiento de la SMO en el terciario temprano y la FVT durante el terciario medio (Flores 1998), de tal forma que posteriormente pudieron establecer zonas de contacto con el evento de glaciación del Pleistoceno (Mayr 1982), ya que tanto los eventos de vicarianza del Neogeno, como los cambios climáticos del Pleistoceno, impactaron de manera importante en la diversificación de P. orbiculare (Bryson et al. 2012).

En cuanto a $P$. o. durangoensis, esta subespecie muestra diferencias significativas $(P=0.024)$ con respecto a $P . o$ orientale; en la gráfica de variación canónica se observan las dos subespecies en los extremos positivo y negativo del eje CVA1 y con la mayor distancia de Mahalanobis (17.18). Horowitz (1955) coloca estas dos subespecies en grupos diferentes, donde el grupo que contiene a $P$. o. orientale presenta la cabeza más amplia. En el presente estudio también se encontró que en la placa fina de deformación esta subespecie presenta la región que corresponde a los cuernos occipitales y temporales más amplia que $P$. o. durangoensis, lo cual coincide con lo reportado por Horowitz (1955). En cuanto a la distribución geográfica, $P$. $o$. durangoensis se encuentra en la Sierra Madre Occidental (SMOc) y, como ya se mencionó, $P$. o. orientale en la $\mathrm{SMO}$; la distribución de estas subespecies hacia el sur se encuentra limitada por la FVT que es una cordillera que emergió recientemente y posiblemente impidió el flujo de estas poblaciones hacia el sur y entre ellas, por lo que quedaron aisladas por la MC. 
El aislamiento de las subespecies mencionadas dio como resultado un impedimento en el flujo de genes y las poblaciones pudieron quedar aisladas históricamente (Bryson et al., 2011). Una evidencia de este hecho es que, con el análisis de ADNmt, las localidades que se distribuyen en la SMOc corresponden al clado Norte y las que se distribuyen en las SMO al clado Sur (Bryson et al., 2012), mientras que con el análisis de morfometría geométrica la subespecie $P$. o. durangoensis que se distribuye en la SMOc y $P$. o. orientale que se distribuye en la SMO muestran variaciones morfológicas evidentes. Resulta claro, por tanto, que los análisis morfológicos del presente estudio concuerdan con lo reportado por Bryson et al. (2012) a partir de análisis de ADNmt. Smith et al. (1997) mencionan que si las subespecies presentan alopatría y, si ya no existe flujo génico entre ellas, podrían ser elevadas a especies, ya que a pesar de que existe un entrecruzamiento potencial entre ellas, la probabilidad es muy baja y el grado de divergencia muy elevado.

Finalmente, las poblaciones de Morelos y el Estado de México que corresponden a la subespecie orbiculare, no mostraron diferencias significativas con ninguno de los análisis morfométricos realizados. Estas poblaciones posiblemente presentan algunas zonas de contacto con $P$. o. cortezii ya que no existen barreras geográficas evidentes que las aíslen, por lo que puede existir flujo génico entre ellas y no se encuentran aisladas completamente. A pesar de que en el estudio de Bryson et al. (2012) no se incluyeron ejemplares del estado de Morelos, si se puede apreciar que las poblaciones del Estado de México y Distrito Federal, se encuentran en un mismo linaje, por lo que, para esta subespecie existe una evidente relación tanto morfológica como genética.

Existe una serie de controversias acerca del concepto de subespecie, desde los autores que consideran este término en completo desuso debido a que su denominación es subjetiva (Mayr 1982), hasta quienes consideran que las subespecies son expresión de los procesos evolutivos y son categorías taxonómicas de gran importancia, que además constituyen modelos que proporcionan información importante de los mecanismos de especiación, siempre y cuando las subespecies se encuentren bien definidas (Monroe 1982). En el presente trabajo, con los análisis de morfometría geométrica se determinó que $P$. o. boucardi no debe ser considerada un intergrado entre $P$. o. orientale y $P$. o. cortezii, así como que $P$. o. orientale y $P$. o. durangoensis muestran variación morfológica significativa. Por lo que, con base en la evidencia de los análisis morfométricos, sí como los resultados del trabajo de Bryson et al. (2012), $P$. o. orientale y $P$. o. durangoensis deberían ser consideradas como especies. Es importante destacar que los análisis de morfometría geométrica permitieron definir claramente estos dos grupos y esta definición no se observa con los análisis de morfometría tradicional.

AGRADECIMIENTOS. Al Dr. Adrian Montes de Oca curador de la Colección de Anfibios y Reptiles del museo Alfonso L. Herrera de la Facultad de Ciencias UNAM, al Dr. Víctor H. Reynoso Rosales cu- 
rador de la Colección Nacional de Anfibios y Reptiles del IBUNAM y al Dr. David Lazcano Villarreal, responsable de la Colección de Herpetología de la Escuela de Biología de la UANL. Por proporcionar las facilidades en el préstamo de los ejemplares que se utilizaron en este trabajo. A Lorena López González, por sus comentarios y apoyo.

\section{LITERATURA CITADA}

Adams, D. C. \& Collyner, M. L. 2007. Analysis of character divergence along environmental gradients and another covariates. Evolution, 61: 510-515.

Bastos-Silveria, C. \& Lister, A. M. 2007. A morphometric assessment of geographical variation and subspecies in impala. Journal of Zoology (London), 271: 288-301.

Bookstein, F. L. 1991. Morphometrics Tools for Landmarks Data: Geometry and Biology. Cambridge University Press, Cambridge.

Bryson, R. W., García-Vázquez, U. O. \& Riddle, B. R. 2012. Diversification in the Mexican horned lizard Phrynosoma orbiculare across a dynamic landscape. Molecular Phylogenetics and Evolution, 62: 87-96.

Flores, V. O. 1998. Herpetofauna de México: distribución y endemismo, pp. 251-278. In: Ramamoorthy, T., Bye, R., Lot, A. \& Fa, J. (Eds.). 1998. Diversidad biológica de México: orígenes y distribución, Oxford University Press. Oxford.

Horowitz, S. B. 1955. An arrangement of the subspecies of the horned toad, Phrynosomaorbiculare (Iguanidae). American Midland Naturalist, 54: 204-218.

Lawrence, P. G., Russell, A. \& Ryan, M. 2002. Ontogenetic scaling of the cranial horn array in Phrynosoma orbiculare (Squamata: Phrynosomatidae). Journal of Herpetology, 4: 578-589.

Manier, M. 2004. Geographic variation in the long-nosed snake, Rhinocheilus lencotei (Colubridae): beyond the subspecies debate. Biological Journal of the Linnean Society, 83: 65-85.

Marcus, L. F. 1990. Traditional morphometrics, pp.77-122. In: Rohlf, F. J \& Bookstein, F. L. (Eds). Proceedings of the Michigan Morphometrics Workshop. Special Publication Number 2. Ann Arbor, Michigan, The University of Michigan Museum of Zoology.

Mayr, E. 1982. Of what use are subspecies? Auk, 99: 593-595.

Méndez de la Cruz, F., O. Hernández Gallegos \& Rodríguez Romero, F. 2003. Phrynosoma orbiculare. Elaboración de fichas de 5 especies de lacertilios: PROY-NOM-059ECOL-2000. Departamento de Zoología, Instituto de Biología, Universidad Nacional Autónoma de México. Bases de datos SNIB-CONABIO. Proyecto W033. México. D.F.

Montanucci, R. 1979. Notes on systematic of Horned Lizards allied to Phrynosoma orbiculare (Lacertilia: Iguanidae). Herpetologica, 35:116-124.

Montanucci, R. 1987. A phylogenetic study on the Horned Lizards genus Phrynosoma, based on skeletal and external morphology. Science, 390:1-36.

Montanucci, R. 2004. Geographic variation in Phrynosoma coronatum (Lacertilia Phrynosomatidae): Further evidence for a peninsular archipielago. Herpetologica, 60: 117-139.

Monroe, B. L. 1982. A modern concept of the subspecies. The Auk, 3: 608-609.

O'Neill, J. P. 1982. The subspecies concept in the 1980's. The Auk, 3: 609-612.

Rasband, W. S. 2009. ImageJ; National Institutes of Health, Bethesda, Maryland, USA, http://rsb.info. nih.gov/ij/, 1997-2007.

Rategar-Pouyani, N. 2005. A multivariate analysis of geographic variation in the Trapelus agilis complex (Sauria: Agamidae). Amphibia-Reptilia, 26: 159-173

Smith, H. M. \& Taylor, E. H. 1950. An Annotated Checklist and Key to the Reptiles of Mexico Exclusive of the Snakes. Bulletin United States National Museum, 199: 1-253. 
Smith, H. 1942. Another case of species versus subspecies. American Midland Naturalist, 1: 201-203.

Smith, H., Chizar, D. \& Montanucci, R. 1997. Subspecies and classification. Herpetological Review, 28: $13-16$

Wilson, E. O. \& Brown, W. L. 1953. The subspecies Concept and Its Taxonomic Aplication. Systematic Zoology, 2: 97-111.

Zelditch, M., Donald, L., Davis, S. \& William, L. 2004. Geometric Morphometrics for Biologists: a primer. Elsevier Academic Press. USA. 443 pp.

\begin{abstract}
ANEXO
Museo "Alfonso L. Herrera” de la Facultad de Ciencias de la Universidad Nacional Autónoma de México; FC00229, FC171, FC230, FC231, FC337, FC602, FC622, FC784, FC02320-4, FC 03104-5, FC 3243-2-SER-2, FC 3243-2-SER-3, FC 03243-3, FC 03530, FC 05871, FC 6010, FC 06245-5, FC 062456, FC 06337-2-3, FC 06337-2-4, FC 06337-3, FC 06337-4, FC 06337-6, FC 08471-3, FC 08471-4, FC 12569-4, FC 12571-3, FC 12572-4, FC12572-5, FC 12597-3, FC 12597-5, FC 12597-7, FC 12598-2, FC 12598-3, FC 12598-4, FC 13788-2, FC 14290-4, FC 14253-3, FC 14523-3, FC 14523-4, FC 21066, FC 21067, FC21068. Colección Nacional de Anfibios y Reptiles del Instituto de Biología, UNAM, IB03133, IB2249-3, IB3169-2, IB3309-4, IB3322-2, IB3625-2, IB5610-3, IB 5681-2, IB 8221-4, IB 9433-2, IB 12451-4, IB 12452-1, IB 12452-4. Colección Herpetológica de la Facultad de Ciencias Biológicas de la Universidad Autónoma de Nuevo León (UANL) UANL1602, UANL2143, UANL1567, UANL3771, UANL-ECB44, UANL1568. Perote, Veracruz (N19³2’31.2’'; W97¹6’38.2’').
\end{abstract}

